\title{
Educación popular, una cosmovisión desde la cotidianidad: Un estudio contextualizado en una comunidad campesina
}

\author{
Popular education, a worldview from everyday life: A contextualized study \\ in a rural community
}

Jorge Moisés Guédez Inojosa

moiseando@hotmail.com

Código ORCID: 0000-0002-1289-8570

Universidad Nacional Abierta, Venezuela
José Pérez Veloz

perezveloz@gmail.com

Código ORCID: 0000-0002-5552-2522

Universidad Nacional Experimental Simón Rodríguez, Venezuela

Recibido: septiembre 2019 / Arbitrado: octubre 2019 / Publicado: enero 2020

RESUMEN

El propósito fue comprender la educación popular desde la cosmovisión de la vida cotidiana de los actores sociales, en el contexto de la comunidad de Palmero, San Carlos, estado Cojedes. Se empleó el enfoque cualitativo, bajo el paradigma interpretativo, método etnográfico. La fiabilidad se obtuvo a través de la técnica de triangulación. Se utilizó para la recolección de datos la observación participativa, el diario de campo y entrevistas. Las conclusiones permitieron evidenciar un contraste vivencial del residente de la comunidad, pues actúa como si no le perteneciera el contexto del cual se beneficia de la actividad agrícola, fuente de sostenibilidad económica practicada como rutina, mediante la tala y quema, sin valorar la importancia hacia la condición humana causando gran impacto ambiental, donde el camino para solucionar el problema es la educación popular, cuyo proyecto implementado por la comunidad desde 1997, no ha tenido apoyo de los entes del Estado venezolano, aun así está dando sus frutos positivos de manera lenta y progresiva.

Palabras clave:

Educación popular; cosmovisión; cotidianidad

ABSTRACT

The purpose was to understand popular education from the worldview of the daily life of social actors, in the context of the community of Palmero, San Carlos, Cojedes state. Methodologically it was a qualitative approach, under an interpretive paradigm, ethnographic method. Reliability was obtained through the triangulation technique. Participatory observation, field diary, and interviews were used for data collection. The conclusions made it possible to show an experiential contrast of the community resident, since he acts as if the context from which he benefits from agricultural activity, a source of economic sustainability practiced as a routine, does not belong to him, without assessing the importance to the human condition causing great environmental impact, where the way to solve the problem is popular education, whose project implemented by the community since 1997, has not had support from the entities of the Venezuelan State, even so it is slowly bearing positive results and progressive.

Keywords:

Popular education; worldview; daily life 


\section{INTRODUCCIÓN}

Históricamente la educación se ha considerado una ciencia importante en la transmisión de conocimientos, formación del potencial humano, el continuo aprendizaje a lo largo de la vida y la socialización. Además como el instrumento que permite a los pueblos y naciones avanzar, pues es el camino hacia la investigación y el desarrollo, en otras palabras, "es praxis, reflexión y acción del hombre sobre el mundo para transformarlos" (Freire, 2009, p.3).

Actualmente, es significativo distinguir la educación popular como un modelo educativo caracterizado por su función incluyente a desertores de los estudios, o población excluida en general. Este modelo, paralelo a la enseñanza tradicional, pero igual impregnado de incontables experiencias exitosas, permite legitimar las prácticas, rutinas, costumbres, es decir, el saber de la gente a través del aprendizaje espontáneo emergido de la intuición emocional.

Ahora bien, conviene definir qué se entiende por educación popular. La misma según Moro (2015) es: “...Un campo específico de acción conformado por diversas prácticas educativas más o menos institucionalizadas: alfabetización, instrucción escolar, formación técnica, animación cultural, formación sindical o política, lectura y animación de textos" (s.n.).

Otras tendencias ubican a esta corriente educativa como un proceso adaptado a las distintas realidades sociales a través de la participación y transformación, donde la cotidianidad y manera de ver la vida es lo que marca el rumbo formado por un cúmulo de vivencias y /o experiencias locales. Experiencia diaria que permite concientizar al educando sobre sus conocimientos prácticos generando resultados positivos, en particular fortalecimiento de las realidades y por ello el poco o nulo apoyo políticoideológico del aparato del Estado. Ciertamente, la cotidianidad da luces para analizar los modos de vida de una comunidad. En ese sentido, Pérez y Pineda (2011) refuerzan lo indicado con sus aportes significativos.

En el seno de la cotidianidad se configura y desconfigura el rostro humano de la sociedad; todo va a depender de los valores que regulen el nivel de cohesión integradora de la comunidad, mediante la acción y el comportamiento de los ciudadanos. Obviamente, las comunidades constituidas sobre la base del respeto, en función del bien común, alcanzan mayor nivel de bienestar y progreso social, que aquellas con baja o inexistente sinergia caracterizadas por la conflictividad, cuyos integrantes actúan más por egoístas intereses individuales que colectivos, sin respetar las normativas sociales. (p.8)

Retomando el ámbito conceptual, la Federación Internacional de "Fe y Alegría" (2015) ajusta la definición de educación popular resaltando que en el marco de

Un proceso histórico y social que, desde la inserción real en el medio popular y en un esfuerzo permanente por ir captando el momento preciso de sus necesidades, tiende a la promoción de las personas y las comunidades para que sean conscientes de sus potencialidades y valores, adquieran la capacidad de decidir sobre su vida y futuro y se constituyan así en protagonistas de su propio desarrollo. (s. n.)

Los autores de la presente investigación coinciden con las definiciones antes señaladas, por cuanto la educación popular es el mecanismo extraordinario de apoyo al desvalido a superarse, permitiendo visualizar debilidades, fortalezas, oportunidades y amenazas desde los contextos comunitarios urbanos o rurales en el sentido amplio del respeto a la condición humana, unido a 
costumbres, valores y formas de vida donde impera el diálogo en ese proceso de relaciones.

Con respecto al tema de estudio, en Latinoamérica destacan una serie de personalidades protagonistas de esta corriente educativa. Es, según Torres (2013):

Una corriente pedagógica que surge a comienzos de los sesenta del siglo XX con la propuesta de educación liberadora impulsada por el Movimiento de Cultura Popular liderado por Paulo Freire en Brasil. Se convierte en un movimiento educativo cuando en las décadas siguientes las ideas de Freire son recreadas en la práctica por miles de educadoras y educadores de base, maestros, animadores culturales y militantes sociales, organizaciones civiles y redes a lo largo y ancho de América Latina. (s.n.)

En consecuencia, la educación popular es una metodología pedagógica bañada de prácticas importantes más allá de las infraestructuras educativas, expandiéndose hacia los contextos socioculturales comunitarios y rurales, potenciando los saberes técnicos de los sujetos en sus ámbitos de interacción diaria, incluyendo también el fortalecimiento del trabajo del campesinado y demás organizaciones sociales y gremiales.

En Venezuela, con respecto al tema, entre los personajes e instituciones más influyentes se encuentran Simón Rodríguez, el maestro Luis Beltrán Prieto Figueroa con el humanismo democrático y la educación social, el profesor Antonio Pérez Esclarín, Prof. Alejandro Moreno con su labor educativa hacia las zonas populares, dando origen a un método de investigación en las ciencias sociales como las historias y relatos de vida, uniéndose el académico José Pérez Veloz, el Movimiento de Educación Popular de "Fe y
Alegría", entre otros, quienes con sus ideas han sido la base de esta corriente educativa.

A título ilustrativo, cuando se habla desde la cotidianidad nacional son significativas las experiencias exitosas con relación al desarrollo y la implementación de programas de educación en los distintos niveles: adolescentes, jóvenes, población adulta, hombres y mujeres bajo la modalidad de educación semipresencial, educación popular emprendida por el Instituto Radiofónico "Fe y Alegría", organizaciones no gubernamentales (ONG), grupos de estudiantes y académicos, con una labor importante hacia las comunidades, contribuyendo a combatir la infravaloración humana cargada del miedo al emprendimiento y superación.

Sin duda alguna, muchas prácticas y riquezas del ser humano en su vida creativa, al respecto Pérez y Pineda (2011) señalan:

La educación espontánea, surge de la
experiencia de la cotidianidad, que
también debe ser valorada en sus
justas dimensiones culturalmente
humanizante y productiva; es el
saber que resuelve la mayor parte de
la problemática social y satisface
las necesidades básicas de las
comunidades; son ideas hechas
realidad. (p.18)

Así pues, esta educación permite conocer ese entramado de relaciones comunitarias y por supuesto está inmersa la forma de cómo los miembros de la población ven e interpretan el mundo. De esto proviene el fortalecimiento o mejoramiento de sus prácticas diarias en función de generar cambios positivos y calidad de vida en armonía con la naturaleza. En otras palabras, la definición de cosmovisión se define así: "Manera de ver e interpretar el mundo" (Diccionario Vox, 2014, p.52). Asimismo, Mendoza (2013) señala al respecto: 
Es la percepción que los grupos (...) tienen de las relaciones del Ser con el Cosmos, en un proceso de conocimiento que no está basado en teorías formales, sino en la creencia heredada y transmitida de generación en generación por los miembros del grupo; por eso no es una elaboración filosófica en el sentido tradicional del término. (p.377)

Partiendo de la comprensión e interpretación sobre creencias locales comunitarias, formas de vida, interacciones grupales, se estimó que al final contribuirán a responder mejor los enunciados o interrogantes de la investigación y mediante la educación popular favorecer las realidades sociales comunitarias.

Desde esa perspectiva, el estado Cojedes es una región del centro-occidente del país también cargada de experiencias exitosas en el área de la educación popular, emprendidas desde el Instituto Radiofónico "Fe y Alegría" universidades locales, organizaciones no gubernamentales (ONG), estudiantes y colaboradores con un sentido de responsabilidad, compromiso y mucha mística hacia la labor educativa en favor de los más necesitados.

Con referencia a la comunidad de Palmero, ubicada hacia el territorio norte del municipio San Carlos, Estado Cojedes y para ilustrar al lector sobre el espacio o ubicación geográfica analizada, la investigación se apoya en la infografía como técnica de representación visual, siendo sus coordenadas Latitud: 9.72485, Longitud: -68.6076, en la imagen del mapa satelital.

La cultura comunitaria en estudio no separa la conexión existente entre el hombre (sujeto activo cognoscente) y la naturaleza (objeto pasivo), pues a pesar de maltratarla o degradarla mantiene una relación de vida al servirse de ella para establecer su morada, generando un crecimiento poblacional sin planificación adecuada, cultivándola de rubros agrícolas, criar animales, pescar, cazar y utilizarla de usufructo turístico. Es significativo resaltar que los residentes de esta comunidad son en su mayoría oriundos o nativos originarios de la comunidad, cuya tendencia es continuar residiendo en la misma.

Desde lo ontológico, el Ser de Palmero se inclina en disfrutar de ese medio ambiente colocando en primer punto uno de sus símbolos, el joropo imagen de la "llaneridad" y algunas veces vallenato para deleites musicales en las faenas de trabajo, usa y distribuye el tiempo en juego de bolas criollas y bailes semanales. Conservan prácticas tradicionales como uso del pilón (instrumento hueco de madera) donde trituran el maíz y producir así la mezcla para las arepas o comidas. Las viviendas en precarias condiciones y muchas construidas de barro. También se mantiene la práctica de principios como el respeto y valor hacia el trabajo.

Importante destacar dentro de la descripción comunitaria, en cuanto a las instituciones existentes, cuenta con una Escuela Comunitaria de Educación Ambiental, Ecológica, Agroalimentaria y Turística, surgida desde Proyectos de Educación Popular y con docentes universitarios en su mayoría egresados del mencionado proyecto. Posee una matrícula actual de 33 personas de la zona y comunidades adyacentes brindándoseles apoyo en el trabajo agrícola, nuevas alternativas socioeconómicas (viveros, cría de conejos, elaboración de productos alimenticios artesanales, cultivo de plantas frutales en espacios reducidos, talleres diversos) y formación ambiental.

Asimismo, un Centro de Educación Inicial Bolivariano Ayacucho II, una escuela de primaria con tres aulas que sirven en las tardes de espacio para impartir clases de bachillerato, donde los jóvenes -los motivados- se ven obligados a culminar su enseñanza media fuera de la localidad, existiendo pocos profesionales. Por otra parte, es un espacio donde convergen investigadores de pregrado, aspirantes a doctores y maestrantes, cada quien analiza a la comunidad desde las perspectivas temáticas individuales. Considerando las experiencias educativas antes expuestas, es relevante citar a Pérez y Pineda (2011) 
La educación debe partir de su escenario vivencial, como lo es la familia-escuela-comunidad, que son los espacios en donde se configura el rostro humano, a partir de sus hábitos y valores que marcan el contexto referencial en la cosmovisión del mundo. (p.7)

Sin embargo, a pesar de todas esas potencialidades, y experiencias exitosas socioeducativas de los proyectos de educación popular comunitario, conocimientos transmitidos de generación en generación sobre la visión del mundo, el diagnóstico in situ realizado, refleja que muchos residentes tienen su primaria y secundaria inconclusa; están desanimados, sin ganas de visualizar proyectos de vida, mejorar sus condiciones, predominando el miedo, infravaloración humana y exclusión social.

Se percibe un problema central, el cual es la deforestación y contaminación de la cuenca del Río Tirgua que abastece de agua al $75 \%$ de la población del estado Cojedes "especialmente Tinaquillo, Tinaco y San Carlos". Anteriormente un río abundante en la actualidad su caudal va en franca disminución cargado con muchos materiales y desechos sólidos. Finalmente, a manera de ritual la tala y quema como sistema de trabajo para la siembra en conucos (cultivo de ñame y yuca) en las montañas de manera vertical ocasionando erosión del suelo.

En ese manto de complejidad, la educación popular vía del aprendizaje para fortalecer el desarrollo humano y dignificación de las personas, estimular la toma de conciencia, el impulso del pensamiento crítico y la creatividad, el trabajo socialmente responsable comprometido hacia la protección del medio ambiente y la promoción de otras alternativas socioeconómicas que permitan mejorar la situación problemática.

Por lo tanto, las teorías que sustentan la investigación, parten del armazón de la educación popular, tal como refleja la Unesco (2013):
...Una lectura crítica de la sociedad y de la educación conservadora; una intencionalidad política emancipadora; el rol de los sujetos sociales como protagonistas de su emancipación. (...) como resultado de la historia latinoamericana, vinculada con los procesos socio-culturales y políticos del continente, al ideario de Paulo Freire, la teología de la liberación, las teorías del desarrollo y la teoría de la dependencia. (p.90)

No obstante, del soporte teórico, la investigación se apoya en las teorías fundantes y acude a otras teorías emergentes, según los hallazgos en la fase exploratoria. Desde el punto de vista axiológico se aborda bajo un marco claro de valores formados, respetando al hombre persona social por naturaleza; de examinar el contexto entramado de relaciones donde la complejidad de la Educación Popular tiene su asiento, así epistemológicamente impulsar dicho modelo o promocionarlo bajo nueva iniciativa educativa y posible opción de solución al problema de tipo ambiental, motivar otras alternativas productivas y mejorar la calidad de vida.

Las intencionalidades del estudio fueron: a) Conocer el contexto cultural en el cual se inscribe la educación popular en la comunidad de Palmero; b) describir los significados que los actores sociales le otorgan a la educación popular desde su cosmovisión de la vida cotidiana; c) interpretar las manifestaciones socioculturales haciendo énfasis en las prácticas y experiencias de educación popular existentes en la comunidad de estudio, lo que permitió llegar a comprender la educación popular desde la cosmovisión de la vida cotidiana de los actores sociales.

\section{MÉTODO}

La orientación de la indagación fue cualitativa con paradigma interpretativo; se procuró en la comunidad de Palmero, captar de manera multidimensional el modo de vida de los 
residentes, comportamiento, modelo cultural, percepciones, valoración situacional, en otras palabras, análisis del contexto y la totalidad (globalidad), donde no habrá análisis ni procedimientos estadísticos, sino más bien, una riqueza narrativa para generar la información. Una vez interpretado el fenómeno en términos de los significados aportados por las personas, arrojar conclusiones respectivas y producir el conocimiento.

Acorde a lo expuesto, se utilizó el método etnográfico. En este estudio se convivió con grupos sociales o personas que tenían características comunes, con un cúmulo de creencias que les permitía hacer vida entre sí, en contacto con el medio ambiente.

El escenario lo constituyó el contexto sociocultural y geográfico natural donde se originó la interacción directa entre investigador, la persona o el grupo de residentes en la comunidad Palmero. Se partió inicialmente observando el evento, describiendo características comunitarias, también retomando lazos de amistad entre líderes comunitarios y algunos moradores, quienes facilitaron al investigador el acceso a la información. lógicamente, luego de la planificación, mantener vínculos con el grupo social, recabar información sobre el lenguaje, expresiones, sentimientos, pensamientos, ideas, características de la comunidad, participantes, procesos, a fin de reflexionar y tomar los apuntes de la vivencia, que construye y reconstruyen la cosmovisión de la vida cotidiana de la comunidad en estudio.

La selección de los sujetos del estudio se hizo de manera intencionada y estuvo representada por informantes claves, quienes manifestaron sus experiencias y vivencias en torno al tema de la educación popular desde la cosmovisióncotidianidad. Fueron seleccionados tomando en cuenta características como: tiempo de residencia, conocimiento local - educativo y liderazgo comunitario.

Los primeros informantes, conocedores de la realidad y del contexto, son líderes comunitarios, miembros del consejo comunal, activista ambiental; además colaborador de todas las actividades desarrolladas por los centros de educación universitaria en la zona. El segundo informante es el director de la escuela comunitaria de educación ambiental, ecológica, agroalimentaria y turística "Palmero Boca Toma", egresado también del proyecto de educación popular de "Fe y Alegría".

En ese mismo orden de ideas otro informante clave fue la profesora Carmen Ostos, profesional hoy día gracias al proyecto de educación popular de "Fe y Alegría". El último informante fue la Sra. Felicia Veloz líder popular comunitaria, docente egresada del programa de educación popular de "Fe y alegría". Sobre el particular Ander-Egg (2011), refiere "son personas que poseen información relevante para el estudio e investigación que se piensa realizar". (p.126). Cabe destacar, el investigador tiene confianza, conocimiento y contacto con los informantes, debido a los lazos de amistad mantenidos por mucho tiempo producto de las relaciones personales con compañeros de estudio y participantes en el proyecto de educación popular.

Por tratarse de un estudio bajo el enfoque cualitativo, se utilizó como técnica la observación participativa. Dicha técnica obligó a los autores a compartir experiencias, vivencias con los vecinos y los líderes comunitarios arriba nombrados (informantes claves), a fin de poder recorrer y describir el espacio comunitario. Finalmente, la intencionalidad encaminada a comprender a dicha comunidad, desde la cotidianidad y la cosmovisión de sus miembros. Con respecto a lo antes señalado, se utilizó el diario de campo. Este mecanismo importante sirvió al investigador durante el proceso de observación participante, asentar las notas de cada uno de los hechos o eventos relacionados con el tema de estudio.

La técnica descrita usó, como instrumento de apoyo, la guía de entrevista y marcó el rumbo hacia donde el investigador deseó conocer los aspectos del objeto o problema, pues tuvo las 
preguntas las cuales fueron elaboradas a partir del primer encuentro reflexivo o notas de campo tomadas de las observaciones, lo cual condujo a seleccionar los informantes clave para buscar la información del andamiaje final de la investigación.

\section{RESULTADOS}

Con respecto al primer propósito enfocado a conocer el contexto cultural en el cual se inscribe la educación popular en la comunidad de Palmero, se alcanzaron nuevos conocimientos, pues la indagación mediante el contacto con los actores sociales y el grupo social en cada observación, permitió percibir de cerca el entorno con su proceso productivo, el espacio geográfico, la historia, sentidos de las cosas, conceptos y comportamiento del grupo social.

La cultura fue la base del estudio etnográfico evidenciándose de acuerdo a los actores sociales un gran potencial artístico, talleres de pintura, artesanía, costumbres de la comunidad entre ellos los bailes, y todo lo típico: entre ellas las comidas. Sin embargo, existiendo mucho talento el desarrollo cultural comunitario es insuficiente o escaso, sin existir igualmente políticas públicas y ningún ente rector relacionado al área.

El análisis de la organización comunitaria demostró que cuentan con un consejo comunal y la organización cristiana evangélica. Esto lleva a presumir que los residentes desde su cosmovisión producto de las relaciones diarias no valoran la importancia de la conformación de estructuras sociales en busca del logro de objetivos comunes, tales como mejoramiento comunitario y mejor calidad de vida en sus procesos productivos con rentabilidad económica.

Al abordar los niveles de educación, existe en la comunidad hasta la modalidad de bachiller con menciones adaptadas al contexto cultural bajo una visión de la educación rural.

El recorrido llevó a examinar la estructura familiar: De la familia nuclear extensa muy unida, hasta dos familias dentro de un mismo hogar, ocasionando un crecimiento poblacional y hacinamiento debido a la ausencia del programa de vivienda.

En ese mismo orden de ideas, el acceso al campo permitió indagar acerca de la actividad económica, prevaleciendo en toda la comunidad la siembra de yuca, frijol, quinchoncho, sobresaliendo en mayor porcentaje el ñame como el medio que genera los ingresos económicos producto de la venta. En consecuencia, existe una agricultura de subsistencia que sirve únicamente para sostener al grupo familiar utilizada de generación en generación a través de conocimientos heredados.

La actividad agrícola fuente de sostenibilidad económica y familiar se practica como rutina o costumbre mediante la tala y quema sin valorar la importancia hacia la protección del medio ambiente causando gran impacto ambiental, cuyos efectos derivan en la erosión del suelo y contaminación del rio y del aire.

En la descripción comunitaria se estudió la categoría turismo, por ser una zona muy visitada en diferentes épocas del año, donde el Río Tirgua representa el principal atractivo turístico. El excursionista es el mayor beneficiario principal protagonista al instalarse en tiempos de temporada y obtener ingresos económicos producto de la venta, situación desfavorable para la comunidad por la resistencia al cambio de los residentes, reconocimiento de la pobreza al sostener que no tienen las condiciones para surgir y además debido a la dependencia del Estado protector, es decir, esperan de la inversión oficial.

Los resultados mostraron que los residentes no tienen claridad en el significado de sentido de pertenencia, pues al no darle importancia a la condición humana lógicamente incide en la degradación del medio ambiente o el trato hacia la naturaleza y hacia sus atractivos turísticos como se explicó en líneas anteriores. Aun así, reconocen que están destruyendo el entorno o medio ambiente con la disposición de reflexionar y cambiar sus modos de vida a través de la educación. Se logró evidenciar que la educación 
popular es una modalidad educativa el cual está desarrollando actividades de capacitación en áreas rurales y la formación de talento humano, mediante el trabajo como en familia con la incorporación de estudiantes de pregrado de la Universidad Experimental "Simón Rodríguez", núcleo San Carlos desde la línea de investigación experiencias educativas y escenarios de aprendizajes y estudiantes de postgrado de otras universidades.

Importante destacar las experiencias exitosas o significativas en cuanto a la educación popular, empezando por la creación y funcionamiento de la escuela comunitaria agroecológica y turística, base de formación y graduación de los propios actores sociales, quienes culminaron su bachillerato gracias al programa del Instituto Radiofónico "Fe y Alegría". Compartir prácticas sobre la protección del ambiente, construcción de viveros para reforestación, artes escénicas, iniciativas para el cambio de los modos de producción por la razón de introducción de nuevos rubros agrícolas, técnicas artesanales en la manipulación de semillas.

De igual manera se comprobó mediante los resultados el trabajo con las escuelas de la comunidad y la promoción del desarrollo endógeno (siembra manualidades, patios productivos) como alternativa familiar hacia la obtención de alimentos. Sin duda alguna se está dando encuentros de saberes, aspirando contribuir al cambio de los modos de vida a través de planificación agrícola y diversificación de cultivos.

Otra experiencia positiva la constituye el funcionamiento del Liceo Escultor "Demetrio Silva" con modalidad de estudio a graduar bachilleres en mención agrícola, ecológica y de protección ambiental, donde la cosmovisión del futuro ha llevado a realizar proyecto de creación de una universidad. Igualmente, resalta la innovación popular a través de la pintura ecológica con pigmentos extraídos de la naturaleza (piedras, lajas, arena) facilitándose un aprendizaje significativo, donde los residentes son los constructores activos de su realidad y experiencias de retroalimentación.

No obstante, viendo el cúmulo de experiencias positivas de la educación popular, los actores sociales coinciden en las debilidades o limitaciones de esta modalidad educativa, representada en la falta de recursos económicos y de una buena infraestructura, cuyo mejoramiento visualizan es mediante la intervención del apoyo oficial. Esto da pie para afirmar que una parte de los residentes desconocen o no valoran su propia capacidad de resolver las cosas, al esperar la ayuda del gobierno de turno traduciéndose dicho hecho en una dependencia del Estado protector, el cual conociendo esa futura ayuda en una larga espera, un pequeño grupo decidió firmar un acta de compromiso con miras a visualizar y ejecutar un proyecto en tres años, que sirva para proteger el Río Tirgua, capacitar a la comunidad y crear la red cafetalera de la zona norte del municipio San Carlos.

Posteriormente, se comprobó en cuanto a las creencias que comparten, la manifestación religiosa más numerosa se inclina hacia la religión evangélica, el resto de la población bajo porcentaje por la fe católica quienes veneran a San Rafael Arcángel y al Dr. José Gregorio Hernández; sin embargo se ha debilitado parte de la costumbre religiosa católica al disminuir o desaparecer algunas ritos como las procesiones antes realizadas. Otra parte de los residentes mantiene neutralidad sin participar en ninguna congregación religiosa.

Dentro de las interacciones personales, la historia juega un papel relevante dentro de la vida cotidiana de los residentes al suministrar la dimensión temporal de los hechos ocurridos, al comparar la situación actual política-económica en crisis con los gobiernos pasados, convirtiéndose el tema político en parte de sus conversaciones diarias, así como la manifestación de las necesidades más sentidas a través del lenguaje coloquial, donde reconocen la pobreza por las 
carencias y por la ausencia de políticas públicas agrícolas de apoyo o de ayuda por oportunismo político, sin tener claridad sobre su accionar futuro. Por lo tanto, se repite en cada una de las opiniones la dependencia hacia el Estado protector, ocasionando en algunos productores desmotivación.

Los residentes de la comunidad de Palmero, distribuyen el tiempo en las labores agrícolas desde muy temprano en horas de la mañana antes horas del mediodía, la ama de casa se dedica a labores domésticas, visitas entre vecinos. Se mantienen los juegos tradicionales, tomar cerveza para compartir historias y experiencias, bailar, jugar bolas criollas, el gusto hacia la dulcería criolla. Se une a la recreación el género de la parranda convertida en costumbre, con canticos para celebrar la navidad, conmemoran el carnaval y semana santa.

Por otra parte, acerca de los valores, a pesar de la crisis moral de la sociedad y donde la comunidad estudiada no escapa, algunas veces la baja autoestima que perciben los autores, todavía en gran porcentaje, los residentes de la comunidad conservan, una vocación solidaria, receptividad comunitaria con expectativas de reciprocidad en la calidad de vida.

En resumen, existe un contraste vivencial del residente de la comunidad, pues la mayoría de las personas quieren vivir en la comunidad y aspiran que sus hijos estudien, situación que no concuerda con el trato a la naturaleza por el impacto ambiental causado, contribuyendo al calentamiento global y contaminación del río responsable de suministrar el agua potable al 75\% de la población del estado Cojedes (Instituto Nacional de Estadística 2010). En consecuencia, el residente actúa como si no le perteneciera el contexto del cual se beneficia.

La educación popular presenta debilidades. Una es el escaso apoyo para inaugurar el tecnológico universitario agroecológico y turístico como campo experimental con actividades hacia los niños y residentes en general para proteger la zona, porque solamente con educación se puede resolver el problema.

\section{CONCLUSIONES}

Como primera observación, se destaca que el proyecto de educación popular implementado en la comunidad de Palmero, desde el año 1997, está dando sus frutos positivos de manera lenta y progresiva, sin apoyo de los entes del Estado venezolano. De allí, la necesidad de buscar los mecanismos necesarios para que el sector oficial y privado valoren esta modalidad de estudio como vía de mejoramiento de la condición humana.

Se sugiere, desde el deber ser, la promoción del potencial turístico concatenado con la impartición de talleres sobre formación de futuros empresarios y en actividades de artesanía que permitan atraer al turista, además en talleres sobre la elaboración de bloques de adobe con el fin de mejorar las viviendas o construir posadas.

Se propone se retome de manera permanente la realización y fortalecimiento de talleres de sensibilización y conservación del medio ambiente. Además de implementar un programa de capacitación sobre diversificación de producción agrícola a fin de disminuir en los residentes la dependencia de actividades económicas de subsistencia.

Por último, solicitar la creación y funcionamiento de una oficina o dependencia pública que permita aprovechar el talento y/o potencial comunitario, con el objetivo de lograr un desarrollo cultural entre los pobladores. Presentar a las autoridades proyecto de construcción de la universidad o tecnológico de educación popular, agroecológica, protección ambiental y turística con actividades destinadas hacia los niños y residentes de la zona estudiada. 


\section{REFERENCIAS}

Ander-Egg, E. (2011). Aprender a investigar: Nociones básicas para la investigación social. Córdoba, Argentina: Brujas

Diccionario Vox. Secundaria y bachillerato, lengua española. (2014). (3a reimpresión). Barcelona: Larousse

Federación Internacional Fe y Alegría. (2000). Educación Popular y Promoción Social. Propuesta de Fe y Alegría. Caracas: Autor

Freire, P. (2009). La Educación como práctica de la Libertad. España: Siglo XXI

Mendoza, M. (2013). El desarrollo local complementario. (Un manual para la teoría en acción, Versión digital). La Paz, Bolivia

Moro, W. (2015) Educación Popular: Un acercamiento a una práctica libertaria. Recuperado de URL: http://www.nodo50.org/pretextos/educ1.htm

Organización de las Naciones Unidas para la Educación, la Ciencia y la Cultura (Unesco) (2013). Aportes conceptuales de la educación de personas jóvenes y adultas: hacia la construcción de sentidos comunes en la diversidad: Autor

Pérez, J., y Pineda, C (2011). Una visión educativa desde la dignidad humana. Revista electrónica encuentro transdisciplinar 1, 18

Quijano, G, y Asselborn, E. (2012). Introducción a la Filosofía y Antropología Filosófica. Documento de cátedra del Departamento de Derecho de la Facultad Teresa de Ávila de la Universidad Católica Argentina. Disponible en: http://bibliotecadigital.uca.edu.ar/repositorio/ contribuciones/abogacia-filosofia-

antropologiafilosofica.pdf

Silva, C. (2012). Aproximación etnográfica a la educación popular liberadora desde la concepción de Fe y Alegría. Trabajo especial de maestría no publicado. Universidad de Carabobo

Torres, A. (2013). La Educación Popular latinoamericana: contextos y desafíos actuales. Recuperado de http://www.revistapueblos.org/?p=14114 